\title{
A cognitive framework based on deep neural network for classification of coronavirus disease
}

\author{
Sapna Kumari ${ }^{1} \cdot$ Munish Bhatia ${ }^{2}$ (i)
}

Received: 26 November 2021 / Accepted: 31 January 2022

(c) The Author(s), under exclusive licence to Springer-Verlag GmbH Germany, part of Springer Nature 2022

\begin{abstract}
Since December 2019, the pandemic of coronavirus (CorV) is spreading all over the world. CorV is a viral disease that results in ill effects on humans and is recognized as public health concern globally. The objective of the paper is to diagnose and prevent the spread of CorV. Spatio-temporal based fine-tuned deep learning model is used for detecting Corv disease so that the prevention measures could be taken on time. Deep learning is an emerging technique that has an extensive approach to prediction. The proposed system presents a hybrid model using chest X-ray images to early identify the CorV suspected people so that necessary action can be taken timely. The proposed work consists of various deep learning neural network algorithms for the identification of CorV patients. A decision model with enhanced accuracy has been presented for early identification of the suspected CorV patients and time-sensitive decision-making. A SQueezeNet model is used for the classification of the CorV patient. An experiment has been conducted for validation purposes to register an average accuracy of $97.8 \%$. Moreover, the outcomes of statistical parameters are compared with numerous state-of-the-art decision-making models in the current domain for performance assessment.
\end{abstract}

Keywords Deep learning $\cdot$ Convolutional neural network $\cdot$ Transfer learning $\cdot$ Coronavirus

\section{Introduction}

Healthcare is a very substantial issue to be managed effectively for any country's growth. One of the major challenges for any healthcare agency is to protect the people from diseases that can be spread from one infected person to another through the air or other communication medium. A novel Coronavirus (N-CorV) is one of the common virus infection diseases which was firstly originated in Wuhan city (China). The Emergency Committee of the World Health Organization (WHO) on January 30, 2020, declared coronavirus as a pandemic as it spreads rapidly from person to

Munish Bhatia

munishbhatia90@gmail.com

Sapna Kumari

sanagupta1986@gmail.com

1 Research Scholar, Department of Computer Science and Engineering, Lovely Professional University, Phagwara, India

2 Assistant Professor Department of Computer Science and Engineering, Lovely Professional University, Phagwara, India person and most affected to the people with a weak immune system $^{1}$. On February 11, 2020, the WHO designated the 2019-nCoV epidemic disease as Coronavirus Disease 2019 (CorV) (Gorbalenya et al. 2020). Severe Acute Respiratory Syndrome (SARS) CoV-2 is a new type virus of novel coronavirus family (N-CorV) (Lai et al. 2020; Stoecklin et al. 2020). N-CorV is categorized under the family of coronavirus which can cause multiple diseases in humans as well as animals. The N-COV-19 pandemic as of date has $306,173,517$ confirmed cases with more than a thousand deaths. Coronavirus symptoms are similar to moderate Middle East Respiratory Syndrome (MERS) or Severe Acute Respiratory Syndrome (SARS). The N-CorV is characterized as a serious threat owing to its higher death rate and increased medical problems. The advent of prevailing computing and emerging technologies can help make a powerful health monitoring system in a real-time application to slow down its spread. In 2003, SARS viral respiratory disease associated with coronavirus was found in South Arabia. In 2015, the MERS outbreak was detected in Saudi Arabia and caused 858 deaths. A CorV patient may have a variety of

\footnotetext{
1 source:https://www.who.int/emergencies/diseases/novel-coronavirus-2019/events-as-they-happen.
} 
symptoms and signs of infection, including fever, dry cough, kidney failure, and respiratory disease, and throat infection that resulted in severe acute respiratory distress (Salman and Salem 2020). In severe circumstances, the infection can lead to pneumonia, breathing problem, multi-organ failure, and death (Mahase 2020). The health systems of several advanced countries are on the verge of collapsing, as a result of the increasing rate of CorV patients. A sufficient number of testing kits is lacking in many countries throughout the world. Many nations have announced total lockdown and ordered citizens to stay home.

The main communication sources of airborne diseases transmission are air droplets and human contact (Chang et al. 2020). To combat CorV, effective and accurate screening is very important (Wang et al. 2020). The patient can be isolated and home quarantined to reduce the transmission through the contact of that person ( $\mathrm{Li}$ et al. 2020). The main core screening method is a real-time reverse transcription-polymerase chain reaction (RT-PCR) for early detection of CorV (Corman et al. 2020). The test is performed on the patient and the report can be obtained within 6-48 h (Xie et al. 2020). An alternate method to RT-PCR is chest computed scan images (CT) (Adair and Ledermann 2020). Intrinsically, a Deep learning(DL)-based diagnostic approach can encourage the experts to achieve an accurate and rapid decision to diagnose the CorV (Shibly et al. 2020).

\subsection{State-of-the-art contributions}

Based on the aforementioned aspects, numerous research has been published in the literature including the diagnosis of CorV. The current study indicates a model that can separate CorV, viral pneumonia, bacterial pneumonia, and healthy cases (normal) into a 4-class model. Here, the major contributions are listed below:

1. A SQueezeNet model is presented for the rapid diagnostic of CorV incorporated DL approach.

2. An offline augmentation is performed to find the solution to the imbalance problem of the open-source dataset. In addition, the benefits of transfer learning and fine-tuning are incorporated to reduce the problem of overfitting and speed of convergence.

3. To achieve and enhance classification results, the current research focuses on binary and multi-class classification using a pre-trained fine-tuned CNN model.

4. The proposed framework outperformed state-of-the-art decision-making techniques for extracting features by using learned weights from pre-trained $\mathrm{CNN}$ models.

Many studies have been stated that DL approaches seem to be significant potential for detecting the CorV disease classification. The current study aims to present a deep neural network-based model for the early detection of CorV. Inspired from the beneficial aspects of a CNN-based deep neural network-based SQueezeNet model incorporates dynamic graph-based spectral clustering using a multi-feature extraction strategy is used for the accurate detection of CorV. Additionally, the proposed model utilizes fine-tuned DL technique on a limited dataset to avoid the overfitting problem. Pre-trained CNN models of Inception-ResNetV2, GoogleNet, VGG2, ResNet152, AlexNet, and DenseNet512 were applied as transfer learning models to detect CorV from chest X-rays images. Henceforth, the suggested model can be further used for large datasets with the benefits of transfer learning and fine-tuning.

The following are the key aspects of the proposed study;

1. Without manual interventions, the suggested model has an end-to-end structure for selection and classification.

2. The present work has been performed on a large dataset to overcome the problem of overfitting.

3. The proposed model uses six different pre-trained CNN models to outperform results. The performance of the suggested model has significantly higher.

4. The presented model identifies the suspected CorV patients with maximal accuracy so the necessary treatment can be provided timely.

5. Experiments have shown the enhanced results in terms of statistical parameters.

\subsection{Paper organization}

The rest of the paper is structured as follows: Sect. 2 represents the literature work in the current domain of study. Section 3 discusses the proposed model for CorV detection. Section 4 presents the experimental simulation for performance assessment. Finally, Sect. 5 concludes the paper with future research directions.

\section{Related work}

\subsection{DL-based CorV identification techniques}

DL techniques are a subset of Machine Learning (ML) that has significant potential for automatic detection of disease through medical imaging with professional experts. As a result, the medical community has emphasized the need of focusing on the advancement of diagnostic technology (Liu et al. 2019). Jain et al. (2021) used medical images with DL techniques for diagnosing lung-related problems. Furthermore, the authors compared 3 DL models for performance assessment. Afshar et al. (2020) suggested a framework 
model based on Capsule Networks for using X-ray images to diagnose CorV disease. Several convolution layers and capsules are used in the proposed work to remove the imbalance problem of a class. Further, the authors have shown that CorV-CAPS performs satisfactorily on lesser trainable parameters in experimental investigation. Apostolopoulos and Mpesiana (2020) used an evolutionary neural network to identify CorV automatically. The procedure is known as transfer learning, in particular, has been implemented with a neural network to classify into common pneumonia, CorVinduced pneumonia with an achieved accuracy of $93.4 \%$. Hemdan et al. (2020) created a COVIDX-Net model that takes X-ray pictures into account. The COVIDX-Net model was trained using $50 \mathrm{X}$-ray images with seven distinct $\mathrm{CNN}$ models. Zhang et al. (2020) presented a general DL approach for automatically extracting and analyzing areas with a high risk of CorV infection. The scientists used a DL-based method to perform a segmentation stage. The contaminated areas were then analyzed and quantified in the CT scan using specified metrics. Xu et al. (2020) proposed a pre-trained Convolution Neural Network extract a potentially infected portion from the computed tomography images. These regions are used to classify the disease into three categories of CorV, Influenza-A viral, and infection. The experiment result has shown an overall accuracy of $86.7 \%$. Nayak et al. (2021) compared the eight pre-trained models of convolutional neural networks. The findings reveal that ResNet-34 outperformed as compared with other state-of-the-art models for the categorization of CorV from normal instances, with an accuracy of $98.33 \%$. Recent researches have been shown the capabilities of CNN to solve challenging tasks such as classification, segmentation, and object detection.

\subsection{Smart medical data analysis}

Smart medical analysis is an important research domain. Numerous researchers have contributed significantly for healthcare data analysis for real-time services, medical report generation (Huang et al. 2021; Pahwa et al. 2021), and recommender systems. Chan,Zeng,Wu andSun (2018) described detection of numerous abnormalities in the small medical image to detect pneumothorax. The authors used the SVM method to diagnose the features of lung diseases using pattern analysis methods. The authors employed texture segmentation to represent the defective lungs in the proposed detection model. Bai et al. (2020) discussed the CorV Intelligent Diagnosis and Treatment Assistant Program based on the Internet of Things (IoT). The main goal was to enable different levels of CorV diagnosis and treatment by using medical technology. Yildirim et al. (2019) proposed a framework for the diagnosis of diabetes mellitus using the transfer learning approach. Dorj et al. (2018) proposed a model for the classification of skin cancer using SVM and a deep convolutional neural network. Brunetti et al. (2019) discussed a computer-aided system that can support physicians in classifying different kinds of breast cancer, liver cancer, and blood tumors revealed by images. Furthermore, the authors purposed a framework for three kinds of diseases classification acquired via Computer Tomography, Magnetic Resonance, and Blood Smear systems. In the context of computer vision, Zhou et al. (2019) proposed a multi-feature extraction approach with an adaptive graph learning model for unsupervised Reidentification for non-textual data. Furthermore, the authors incorporated multi-feature dictionary learning and adaptive graph learning into a unified learning model. The optimization algorithm is used to prove convergence. The experimental has been performed on four datasets to depict the superiority and effectiveness of the proposed method. Alqudah, Qazan and Alqudah (2020) used the Xception architecture to detect CorV infection by classifying images into three categories. Ucar and Korkmaz (2020) proposed an intelligent and efficient decision-making system for CorV. The authors used an AI-based model incorporating a Bayesian network that outperforms for identification of CorV. Li et al. (2018) proposed an optimization algorithm that is adopted to effectively solve the clustering problem by using one parameter in the learning process. Chang et al. (2015) proposed a novel compound rank-k projection algorithm for bilinear analysis. In the proposed algorithm, the authors used the multiple rank-k projection models to find the best optimization solution. Luo et al. (2017) proposed an unsupervised feature selection to produce a faithful subset from the feature space by maintaining the intrinsic structure accurately. To achieve the subset, optimal graph reconstruction and selective matrix techniques are used. Furthermore, the various results have shown the effectiveness of the proposed algorithm. Ren et al. (2021) discussed the characteristics of the neural search for medical data, issues and provided the comparative analysis for performance assessment. Yu et al. (2018) proposed the Adaptive Semi-supervised Feature Selection (ASFS) for cross-modal retrieval. In the proposed model, the authors used an efficient joint optimization algorithm to update the mapping matrices and label matrix for non-labeled data. Experimental results have shown the reliability and efficiency of the model. Luo et al. (2017) proposed a novel semi-supervised feature analyzing framework for video semantic identification. In the paper, the authors have included the adaptive optimal similarity matrix learning into the procedure of feature selection. In Li et al. (2019), by including adaptive optimum similarity matrix learning into the feature selection technique, authors have created a semi-supervised feature analysis framework for video semantic identification. Moreover, authors have been shown fine-tuned parameters with other methods to 
Table 1 Comparative analysis with state-of-the-art work

\begin{tabular}{|c|c|c|c|}
\hline Authors & Models & Strength & Weakness \\
\hline Panwar et al. (2020) & ncovnet & $\begin{array}{l}\text { VGG-16 used convolutional layers and five } \\
\text { dense layers are integrated in the architecture }\end{array}$ & $\begin{array}{l}\text { As the training loss value is still decreasing before } \\
\text { it is completed. Moreover, Until the network } \\
\text { converges, it is not trained }\end{array}$ \\
\hline Sethy and Behera (2020) & ResNet50 & $\begin{array}{l}\text { Uses ResNet-50 as the feature extractor and } \\
\text { SVM for the classification }\end{array}$ & $\begin{array}{l}\text { Authors uses only } 25 \text { number of images for classi- } \\
\text { fication and also not use the end-to-end network }\end{array}$ \\
\hline Mahmud et al. (2020) & CovXNet & $\begin{array}{l}\text { Multi-dilation convolutional layers are used, } \\
\text { with group convolution performed }\end{array}$ & $\begin{array}{l}\text { After } 45 \text { epochs, training convergence is highly } \\
\text { unstable. The only fact is that each class has only } \\
305 \text { images, making over-fitting easy to detect }\end{array}$ \\
\hline Loey et al. (2020) & ResNet & $\begin{array}{l}\text { To create synthetic data, it uses a deep convolu- } \\
\text { tional generative adversarial network }\end{array}$ & $\begin{array}{l}\text { The study does not produce unique synthetic data } \\
\text { and not trained the network separately for each } \\
\text { class }\end{array}$ \\
\hline $\begin{array}{l}\text { Abdani,Zulkifley and } \\
\text { Zulkifley (2020) }\end{array}$ & SPP-CorV-Net & $\begin{array}{l}\text { The authors have applied a pooling module to } \\
\text { extract deep features }\end{array}$ & $\begin{array}{l}\text { For the experiment includes few images for class } \\
\text { detection performance of CorV cases }\end{array}$ \\
\hline Proposed Model & SQNet & $\begin{array}{l}\text { In the study, employs a data augmentation } \\
\text { through a conditional deep convolutional } \\
\text { neural network and uses fine-tuning technique } \\
\text { to overcome the problem of overfitting }\end{array}$ & create robust model for real-case Application \\
\hline
\end{tabular}

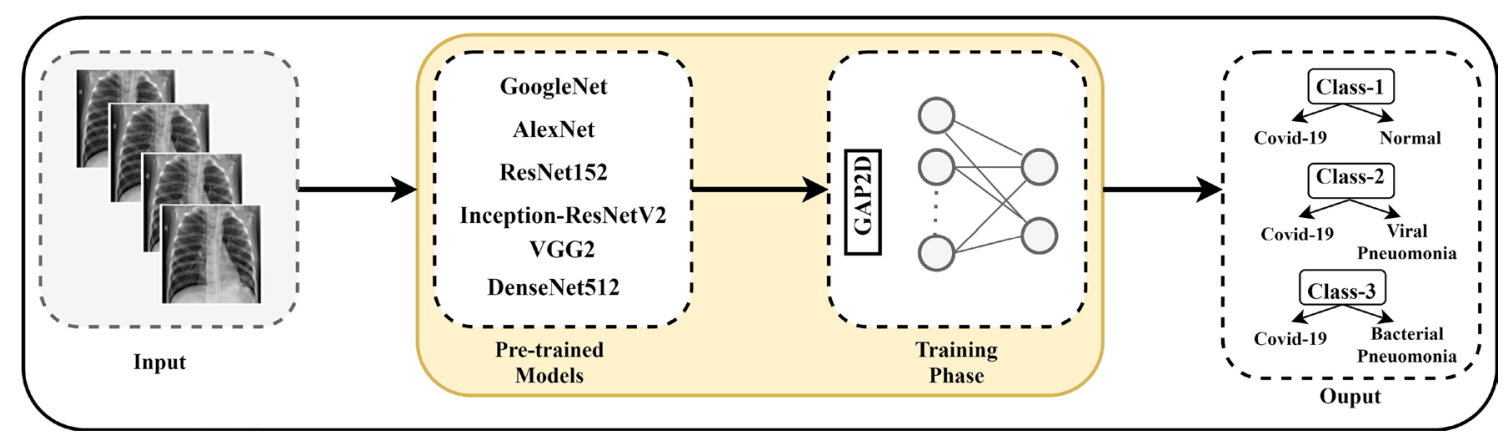

Fig. 1 Conceptual perspective of proposed model

achieve higher accuracy. Table 1 shows strengthen and weaknesses for some of the relevant models presented are numerous researchers.

\section{Proposed model}

DL models have been effectively employed in a wide range of fields, including medical data classification, segmentation, and lesion identification (Brunetti et al. 2019). DL models are used to analyze medical imaging modalities such as magnetic resonance imaging (MRI), computed tomography (CT), and X-ray images (Gaál G et al. 2020). Consequently, studies with the identification of illnesses such as diabetes, brain tumors, and cancer are available. Nowadays, Convolutional Neural Networks (CNNs) model is used to solve image identification and classification issues (Albawi, Mohammed and Al-Zawi 2017). The X-ray images are preprocessed by augmentation and normalization before passing to the fully connected layers of the CNN model. Specifically, images are converted to matrix format. Based on disparities in images and matrices, the system decides which image corresponds to which label. During the training phase, it learns the effects of variations on the labeled data and predicts to generate the new image. Figure 1 depicts the outlined architecture of the proposed model. For such purposes, deep CNN is employed containing 3 different layers: Convolution layer, Pooling layer, and Fully Connected layer. Both convolution and pooling layers are used in the feature extraction process. These layers explicitly assume that any input to them is an image that helps to increase efficiency. Moreover, such layers are effective to extract spatial and temporal features of an image by incorporating filters. Unlike traditional feedforward, these layers contain a considerably reduced number of parameters and employ a weight-sharing and data augmentation approach to reduce the computing requirements (Ahmed, Hossain and Noor 2021). In the proposed work, a Spectral Clustering (SC) approach is used with the proposed 
algorithm to extract clusters values from the input datasets (Li et al. 2018). The pre-processing and feature extraction of all database images are performed for indexing purposes. Each of the layers is detailed ahead;

\subsection{Convolution layer}

The first layer is responsible for identifying numerous attributes of the presented $\mathrm{CNN}$ technique. The learning parameters of each layer consist $3 \times 3$ or $5 \times 5$ shaped matrix kernel which is used to transform the input matrix. When an image is forwarded through a filter, the value from filters creates a feature map of that filter. By applying the filters, such layers are used to extract low and highlevel pattern characteristics. The stride parameter specifies the number of steps to correct for shifting across the input matrix. The output is computed as:

$Z_{j}^{k}=f\left(\sum_{m=1}^{N} w_{j}^{k-1} * y_{m}^{k-1}+b_{j}^{k}\right)$

where, $Z_{j}^{k}$ is feature map in a layer.

$w_{j}^{k-1}$ indicates the jth filter in $\mathrm{k}-1$ layer.

$y_{m}^{k-1}$ represents the feature map in k-1 layer.

$b_{j}^{k}$ indicate the bias of $j^{\text {th }}$ feature map in $k^{\text {th }}$ layer. $\mathrm{N}$ is the total number of features in $(k-1)$ th layer.

$\left.{ }^{*}\right)$ represents vector convolution process. The complexity of proposed algorithm per time step can be expressed as the addition of the complexity of the convolution layers and the pooling layer for all training process. Asymptotically, it is represented as:

$$
\mathrm{O}\left(\left(\sum_{k=1}^{d}\right)\left(s_{k}^{2} * n_{k} * m_{k}^{2}+w\right) * i * e\right)
$$

where $i$ is the input length, $d$ is the number of convolutional layers, w indicates the number of weights, and e denotes the number of epochs.

\subsection{Pooling layers (PLs)}

After the initial layer, the next layers are PLs which can be used to diminish the spatial size of the representation, number of maps attributes, and parameters of the network. It is done by applying the mathematical computation generated by previous kernels after CNN. It is also responsible to extract dominant features(Bailer, Habtegebrial and Stricker 2018). The current study uses the max-pooling (MXP) and Global Average Pooling (GAP) for reducing dimensionality and complexity. The MXP algorithm selects the highest value in each feature map by utilizing the matrix size to give fewer output neurons. MXP layer is generally included between two convolution layers which separate the input and generate the highest value as output.
In addition, the GAP layer finds the average to reduce data into a single dimension. The flattering layer of PL gathers data into a single vector and forward it to the fully connected layer.

\subsection{Fully connected and activation function layer (FCL)}

FCL is the final layer of the presented CNN model. Rectified linear unit (ReLU) activation function is used on the FCL which is similar to multilayer perceptron. Moreover, softmax activation function is utilized to predict the output image. Both these functions are mathematically represented as:

$\operatorname{ReLU}(z)= \begin{cases}0, & \text { if } \quad z<0 . \\ z, & \text { if } \quad z \geq 0 .\end{cases}$

$\operatorname{Softmax}\left(z_{i}\right)=\frac{e^{x_{i}}}{\sum_{j=1}^{c} e^{x_{j}}}$

where, $x_{i}$ represents the inputs and $\mathrm{c}$ indicates the number of classes.

Definition 1 Transfer learning (TL) The process of transfer learning from one pre-trained model to create a new model by using the computed network layer weights is called TL (Swati et al. 2019).

TL technique is more useful in medical applications. The main advantage of adopting the TL approach is that as it allows for data training with fewer datasets and requires less cost in terms of calculations. TL approach in conjunction with deep CNN is used to achieve the information by the pre-defined models, transferred on a large dataset to the model to be trained (Chouhan et al. 2020). Therefore, TL is used to leverage the knowledge such as features, and weights learned from the source domain for training newer models of the destination domain.

\subsection{Stepwise approach}

Figure 2 depicts the step-by-step layered architecture of the proposed model. Some of the brief aspects of the presented technique are mentioned ahead.

1. As convolution is a gradual process, it is applied recursively to an image. It extracts various features of an image as an input.

2. Apply pooling recursively on all the images to make the features robust against noise and dimensionality reduction. 


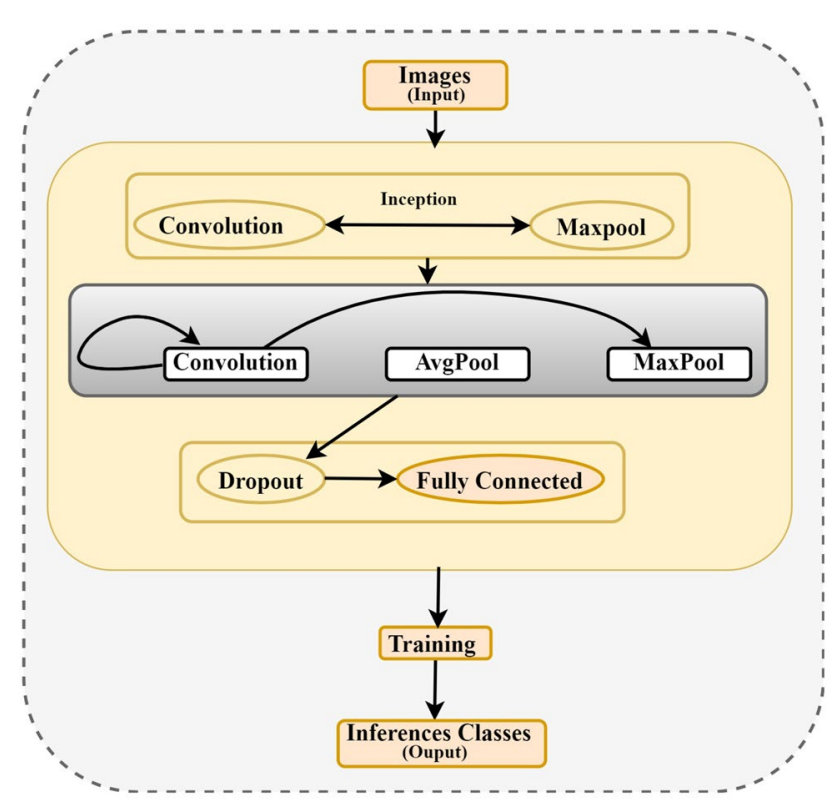

Fig. 2 Layered architecture of proposed work

3. The image is classified according to the labeled training class.

Furthermore, a transfer learning technique is used with the presented CNN model to overcome insufficient data and training delay.

\subsection{Model development}

A convolutional network consists of a chain of convolutional layers, ReLU activation functions, pooling layers, and a fully connected layer with a softmax activation function. Every neuron of each layer is linked to all neurons in the following layer, making CNN a coordinated form of multilayer perceptrons. Convolutional filters increase the visual field when layers convolute input using kernels. The hierarchical process provides high-level feature maps, variation in subsequent input layers, and enhances the performance as it uses a condensed network that helps to train models and reduced computation complexity. The last layer of the proposed model is fully connected and trained with the size of separate output classification classes. The feature maps are used to represent a global state of a network. Pre-trained deep neural networks models are initially trained by using its labeled ImageNet dataset. Therefore, by considering such characteristics, a fine-tuned DL (FTDL) model of SQueezeNet along with CNN is proposed to accomplish the classification process.

\subsection{Decision visualization}

The proposed technique takes into account the visualization of internal representations, feature extraction in convolutional layers, and computational performance as major aspects. The main aim of the presented work is to provide visualization to each convolutional kernel, find out multifeatures without redundancy, and learn to extract features from the visual input when trained with pre-defined CNN models. For such purposes, various feature extraction classification techniques such as spectral clustering can be used for accurate classification. ( $\mathrm{Li}$ et al. 2018) proposed a rankconstrained spectral clustering which is an explicit clustering strategy. The approach utilizes an adaptive neighborhood learning process to find the diagonal affinity matrix of an ideal graph. In the process, the similarity matrix is learned simultaneously. Based on a rank restriction of the matrix, the clusters are guaranteed to converge. The limitation of the explicit clustering is complexity and computational time as it uses the predefined representation. Another approach in Computer-aided vision is zero-shot event detection via the event-adaptive concept. It uses the correlation between an event, concepts, and pre-trained concepts from external sources (Li et al. 2019). Such an event-based concept is more useful in video-based event data. In the present work, the proposed algorithm used dynamic affinity graph construction for spectral clustering using multiple features incorporated CNN for CorV classification (Li et al. 2018). To get an idea about the CorV detection transparency, the current study employed Graph-based spectral clustering (GBSC) using multiple features for detecting the regions where the proposed model has acquired enhanced efficacy for the classification .

\subsubsection{Multifeature spectral clustering (SC)}

The data is effectively transformed into a set of data points in Euclidean space by mapping it into a high-dimensional feature space, where each coordinate corresponds to one of the data items. SC usually constructs an affinity matrix to measure the relationship among data points in space. Such mapping is called the kernel approach which works on CNN layers of the proposed model. The main purpose of SC is to transform the representations of the data points into the indicator space in which the cluster characteristics are more prominent. SC is mainly used in computer vision applications to construct similarity matrices for data partitioning. Consider a data set $\{x\}_{i}^{n} \in R^{d_{m}^{* n}}$ where $d_{m}$ represents the dimensionality of the $m^{\text {th }}$ feature. All data points are grouped into u clusters such that $U_{i} \mathrm{i}=1,2, \ldots$, u such that data points 


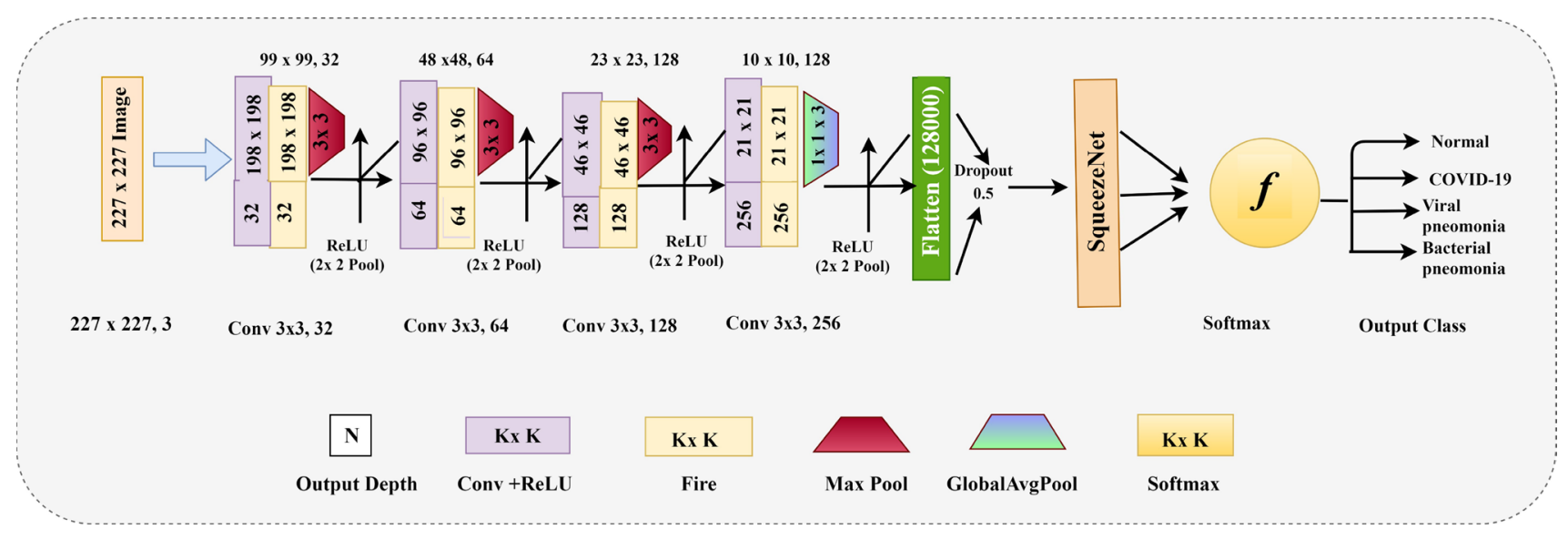

Fig. 3 Architecture of proposed model

from the same cluster can be kept close to one another. Let $\{y\}_{i}^{n} \in R^{d_{m} * u}$ represents the cluster matrix indicators where $y_{i}$ is cluster matrix indicator correspond to $x_{i}$. The scaled cluster matrix is represented as:

$G=Y\left(Y^{T} Y\right)^{-1 / 2}$

The neighbors of $x_{i}$ will be the k-closest samples in a given data set. The weighted affinity matrix can be represented by using the Euclidean distance as:

$p_{\mathrm{ij}}= \begin{cases}\exp \left(-\frac{\left\|x_{i}-x_{j}\right\|^{2}}{\delta^{2}}\right), & \text { if } x_{i}, x_{j} \text { are } k \text { nearest neighbors } \\ 0, & \text { otherwise }\end{cases}$

$\delta^{2}$ is used as a scaling parameter which defines the size of neighbor. The Laplacian graph $\mathrm{L}$ is calculated as $\mathrm{L}=\mathrm{D}-\mathrm{W}$, where $\mathrm{D}$ represents to diagonal matrix as $D_{i i}=\Sigma_{j} P_{i j}$. The normalized Laplacian matrix is written as:

$L_{P}=I-D^{1 / 2} P D^{1 / 2}$

The learned matrix is denoted by $W^{v} \in R^{d_{m} * r_{m}} \cdot r_{m}$ denotes the reduced dimensionality of $\mathrm{m}$ feature at the polling layer. The low dimensional subspace data with constraint $\left(W^{m}\right)^{T} Z_{t}^{m} W^{m}=I$ can de defined as:

$Z_{t}^{m}=\left(X^{m}\right)^{T} E X^{m}$

$Z_{t}^{m}$ is total of scatter matrix.

$X^{m}$ defines the $m^{\text {th }}$ feature of X.

$\left(X^{m}\right)^{T}$ represents the transpose of $X^{m}$.

$E=I-(1 / n) R R^{T}$

where $\mathrm{R}$ is a vector whose all elements are 0 .
All the data points are $x_{i}$ are connected as a neighbour with a probability $W_{i j}$, Then the distance between $x_{i}$ and $x_{j}$ in low dimension subspace is computed as:

$\sum_{m} \alpha_{m}\left\|\left(\boldsymbol{W}^{m}\right)^{T} x_{i}^{m}-\left(\boldsymbol{W}^{m}\right)^{T} x_{j}^{m}\right\|_{2}^{2}$

$\alpha_{m}$ denotes the weight of $m^{\text {th }}$ feature.

As a result, the optimal data similarity matrix is obtained by local learning to generate the optimal affinity graph.

\subsubsection{SQueezeNet (SQNet) model}

SQNet is a convolution network that outperforms AlexNet while using 50x fewer parameters (Iandola et al. 2016). SQNet has eighteen layers, including convolution layers, MXP levels, GAP layer, fire levels, and softmax output layer. Figure 3 represents the architecture of the proposed model. The $\mathrm{K} \times \mathrm{K}$, s, and 1 specify the filter's receptive field size, stride, and length of the feature map respectively. The input has a $227 \times 227$ dimensional image and consists of RGB channels. Convolution is used to generalize the input images and max pooling. With $3 \times 3$ kernels, the weights and small regions in the input are convolved by the first layer of CNN. As the positive component of the argument, each convolution layer executes element-byelement activation. Between the convolution layers, SQNet makes use of the fire layers, which are made up of squeeze and expansion stages. The size $1 \times 1$ filter is used in the squeeze phase, whereas the size $1 \times 1$ and $3 \times 3$ filters are used in the expansion phase. The input tensor $\mathrm{H} \times \mathrm{W} \times \mathrm{C}$ is squeezed, and the total number of convolution channels is equivalent to the number of input tensors i.e.C/4. 


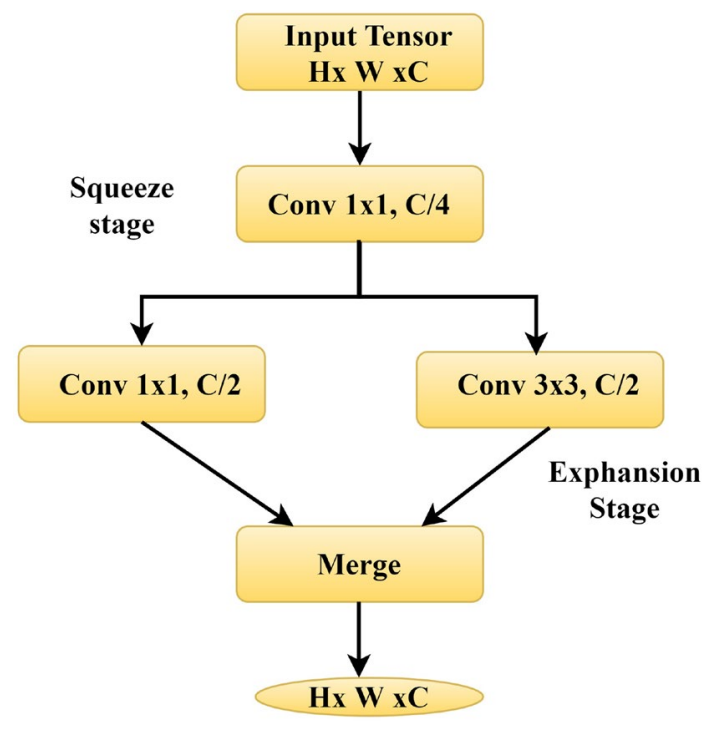

Fig. 4 Summarize structure of fire layer

After the initial step, the data goes through the expansions, and its depth is increased to the output tensor. Both the squeezing and expanding stages involve the ReLU. The squeezing process reduces the depth, whereas the expanded stage increases it while maintaining the same feature size. Finally, using the merging operation, the expanded outputs are stacked in the dimension of the input tensor. Figure 4 depicts all operations of the SQNet architecture.

Finally, the resultant $f(y)$ of the squeezing process with the kernel(W), feature maps(FM), and C specify channels of different tensors respectively, which can be mathematically represented as: $f(y)=\sum_{f m=1}^{F M} \sum_{c=1}^{C} W_{c}^{f} * x_{c}^{f m}$

where $\mathrm{f}(\mathrm{y})$ is an output $\in R^{N}$. Let $X_{i}$ be an input with a size of $\left(W_{i}, H_{i}, C_{i}\right) \in R^{N}$ of layer i. W represent the weights, $\mathrm{H}$ defines the height, and $\mathrm{C}$ indicate the channel respectively. The MXP layers along spatial dimensions execute a downsample in the network and the GAP, which converts the feature map classes into a single value. In the last layer, the Softmax activation function produces multi-class probability distributions. SQNet is a base model along with dropout and FC layers. Table 2 represents the detailed layered, output shape of the model. Multiclass classifiers, which are also known as output layers in Neural networks are often used to classify images into a collection of categories. The classifier requires individual features for the classification to conduct calculations. As a result, for the classifiers, the output given by the feature extractor is transformed into a 1-dimension feature vector. The result of the convolution operation is flattened to produce one long feature vector for the SQNet layer to use in its final classification step, which is known as flattening. A flattened layer, a dropout of size 0.5 , convolution layers, a ReLU, and a softmax activation function perform the classification tasks in the classification layer.

The motivation for developing the SQNet architecture in CorV diagnostic as provides three important benefits:

1. As it employs a lesser number of parameters, the network is more efficient;

2. Applications based on such model is easier to proceed and need less communication; and

3. In context to memory, it is easier to integrate into embedded systems as it requires less than $5 \mathrm{MB}$.
Table 2 Detailed configuration of the model

\begin{tabular}{lllll}
\hline Layer & Layer_Type & Activation & Stride & Output shape \\
\hline Conv_2d & Conv+ReLU & $3 \times 3$ & 2 & $198 \times 198 \times 32$ \\
pool1 & MaxPooling & $3 \times 3$ & 2 & $198 \times 198 \times 32$ \\
Fire & Conv+ReLU(expand+merge) & $1 \times 1$ & 1 & $128 \times 128 \times 32$ \\
& $($ Conv+ReLU(expand+merge))/2 & $3 \times 3$ & 1 & $128 \times 128 \times 64$ \\
pool2 & MaxPooling & $3 \times 3$ & 2 & $128 \times 128 \times 64$ \\
Fire & Conv+ReLU(expand+merge) & $1 \times 1$ & 1 & $56 \times 56 \times 32$ \\
& (Conv+ReLU(expand+merge))/2 & $3 \times 3$ & 1 & $56 \times 56 \times \times 64$ \\
pool3 & MaxPooling & $3 \times 3$ & 2 & $99 \times 99 \times 128$ \\
Fire & Conv+ReLU(expand+merge) & $1 \times 1$ & 1 & $99 \times 99 \times 128$ \\
& $($ Conv+ReLU(expand+merge))/2 & $3 \times 3$ & 1 & $28 \times 28 \times \times 128$ \\
pool4 & MaxPooling & $3 \times 3$ & 2 & $56 \times 56 \times 64$ \\
Fire & Conv+ReLU(expand+merge) & $1 \times 1$ & 1 & $28 \times 28 \times \times 256$ \\
& $($ Conv+ReLU(expand+merge))/2 & $3 \times 3$ & 1 & $14 \times 14 \times \times 256$ \\
Conv_2d & Conv+ReLU & $3 \times 3$ & 1 & $14 \times 14 \times 128$ \\
pool & Global Average Pool & - & - & $1 \times 1 \times 3$ \\
Output & SoftMax & - & - & $1 \times 1 \times 3$ \\
\hline
\end{tabular}


Table 3 Number of images per CorV-Dataset

\begin{tabular}{lllll}
\hline Datasets & Healthy & Bacterial & CorV & Viral \\
\hline Cov-Dataset1 & 2860 & - & 126 & - \\
Cov-Dataset2 & - & 2228 & 126 & - \\
Cov-Dataset2 & - & - & 126 & 3517 \\
\hline
\end{tabular}

4. To initialize the parameters, the current study used TL to solve the problem of overfitting.

\section{Experimental simulation}

DL approaches have been deployed in recent years continue to perform admirably in the domain of medical image processing, as well as in many other fields. It is an endeavor to extract relevant data from medical data by applying DL techniques to it. The study suggested an automated identification of CorV by utilizing deep CNN-based transfer models on chest X-ray images. Transfer learning technique offers a promising technique in case of inadequate data. For such purpose, Inception-ResNetV2, GoogleNet, VGG2, ResNet152, AlexNet, and DenseNet512 are used as pre-trained models to extract learned features. In the current study, to achieve better predictability outcomes for three different datasets, comprising images (X-Ray) of noninfected, infected, bacterial, and viral pneumonia patients are used(Yadav and Jadhav 2019). Furthermore, CNN has proven to be efficient in transfer learning when trained on large-scale datasets like the ImageNet dataset. The collection contains chest X-ray images mostly from patients suffering from SARS, CorV, the Middle East respiratory syndrome (MERS), and pneumonia collected from the GitHub shared by Cohen et al. (2020). In addition, 165 images are selected from the "COVID-chest-ray-dataset" Dataset ${ }^{2}$. The Experiments have been performed on three datasets (Cov-Dataset1, Cov-Dataset2, Cov-Dataset3) with X-ray images. The dissemination of images per dataset is given in Table 3. The collected datasets consist of a total of 8731 images with 7662 patients of disease-infected cases. The data set includes 2860 Normal, 2228 Bacterial pneumonia, 3517 Viral, and 126 CorV disease cases.

In the training dataset, the data augmentation approach is applied with the scaling (1/255), shear range (1/5), zoom (1/5), and horizontal flipping. All images are resized to 227 $\times 227$ pixels in CorV-datasets.

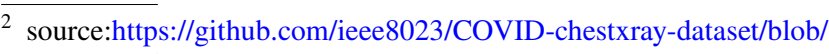
master/metadata.csv.

\subsection{Experimental setup}

The current section describes the experimental setup and performance assessment of the SQNet model. The suggested method's influence is explored in terms of accuracy, evaluation metrics, and computing efficiency. The training and testing are performed in a Matlab environment running on a workstation with $3.3 \mathrm{GHz}$ CPU dual Intel Xeon E5, Quadro M4000 8 GB GDDR6 GPU, and 512 GB of RAM. Furthermore, the evaluation metrics of the proposed network are compared with state-of-the-art techniques.

\subsubsection{Performance evaluation metrics}

The current subsection described the quantitative performance of the proposed approach such as accuracy (ACR), truthfulness (TR), faultless (FLT), specificity (SPFC), f-measure, and statistical measure computed from confusion matrix as Matthew correlation coefficient (MCR) of the proposed model. These metrics are described ahead:

1 ACR determines the classification evaluation of the suggested model.

2 TR specifies the rate of true classification of the images.

3 FLT specifies the corrected detect negative images.

4 F-measure calculates the harmonic mean and the combination of TR and FLT.

5 MCR specifies the classification quality of the performance.

The evaluation matrix as per confusion matrix can be expressed as:

$$
\begin{aligned}
& A C R=\frac{N_{T R P}+N_{T R N}}{N_{T R P}+N_{T R P}+N_{F L P}+N_{F L N}} \\
& T R==\frac{N_{T R P}}{N_{T R P}+N_{F L P}} \\
& F L T==\frac{N_{T R P}}{N_{T R P}+N_{F L N}} \\
& S P F C==\frac{N_{T R N}}{N_{T R N}+N_{F L P}} \\
& M C R=\frac{\left(N_{T R P} * N_{T R N}\right)-\left(N_{F L P} * N_{F L N}\right)}{\sqrt{\left(N_{T R P}+N_{F L P}\right) *\left(N_{T R P}+N_{F L N}\right) *\left(N_{T R N}+N_{F L P}\right) *\left(N_{T R N}+N_{F L N}\right)}} \\
& F-\text { measure }=2 * \frac{T R * F L T}{F L T+T R}
\end{aligned}
$$


Table 4 Raw and augmented datasets class distribution

\begin{tabular}{|c|c|c|c|c|c|c|}
\hline \multirow[t]{2}{*}{ Classes } & \multicolumn{2}{|c|}{ Before augmentation } & \multicolumn{4}{|c|}{ After augmentation } \\
\hline & Train & Validation & Test & Train & Validation & Test \\
\hline CorV & 86 & - & 40 & 1580 & 114 & 113 \\
\hline Normal & 1875 & - & 985 & 1580 & 114 & 113 \\
\hline Bacterial & 1716 & - & 512 & 1580 & 114 & 113 \\
\hline Viral & 2814 & - & 701 & 1580 & 114 & 113 \\
\hline Total & 6537 & - & 2230 & & & \\
\hline
\end{tabular}

Fig. 5 Minimum objective vs function evaluation

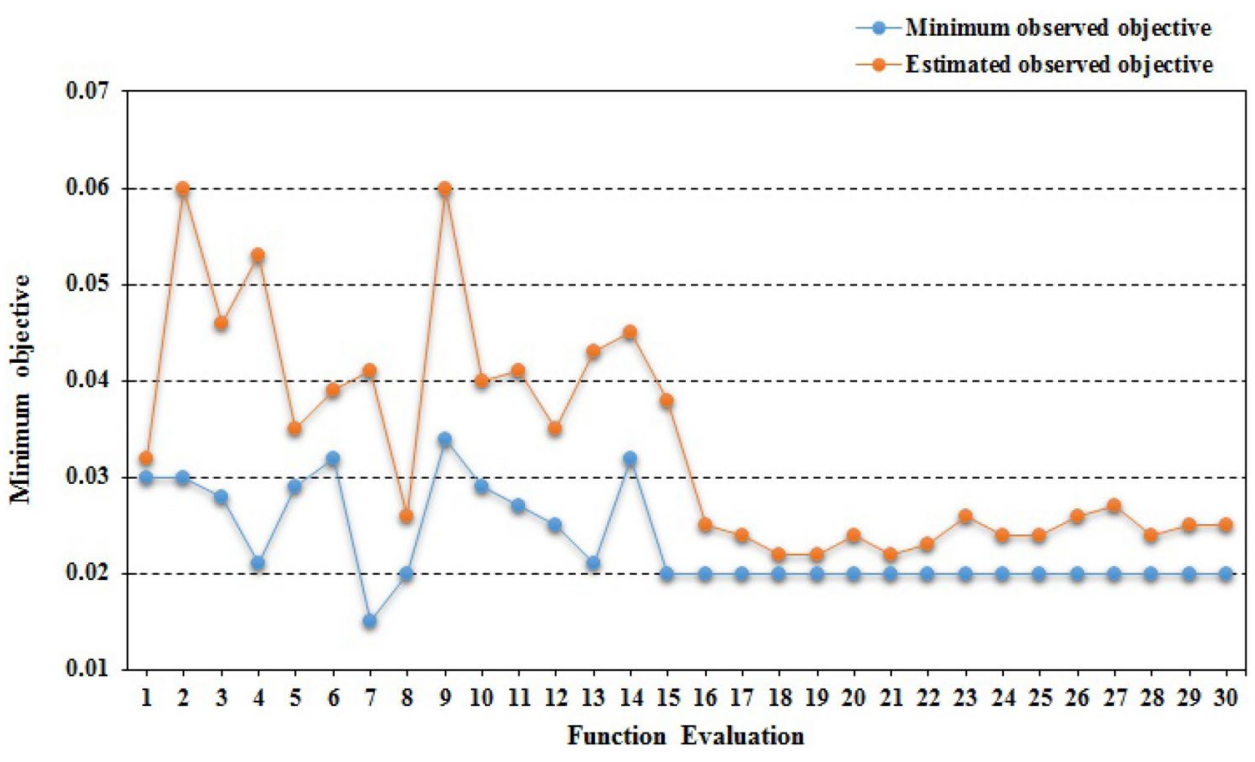

Here $N_{T R P}, N_{T R N}, N_{F L P}, N_{F L N}$ describes the number of correctly classified with the disease, number of correctly defines with another disease, number of incorrectly classified with the disease, number of incorrectly classified with another disease respectively. By using such evaluation metrics, the classification method of the proposed model enhances the performance and effectiveness to determine the disease.

\subsubsection{Training and testing implementation}

Firstly, data augmentation is performed to the raw dataset in the experimental setup both for fine-tuning and end-to-end training. The five-fold cross-validation is used to enhance the validation performance to get a robust result. Further, the enhanced dataset is distributed into training, validation, and testing parts. The dataset packages are split into three groups: $80 \%$ for training, $10 \%$ for validation, and $10 \%$ for testing. The DL SQNet network uses training and validation datasets. The adaptive moment estimation (ADAM) optimizer and the cross-entropy loss function are used for training, with a starting learning rate of 0.001 and decreased by 1 after two epochs. Training accuracy represents a correctly labeled that is shared by the training image. Crossentropy defines that how finely the loss function is trained.
Validation accuracy described the training accuracy. The SQNet has been trained for 100 iterations at a learning rate of 0.001 and subsequently trained at a very low learning rate of 0.0001 . Figure 8 represents the training and testing accuracies and Fig. 9 depicts the loss values for both training and testing. As a consequence of the validation, the objective function error is minimized, and the optimal network model is utilized to implement the testing phase. The derived best model is evaluated using a different test dataset. All of the input images are scaled into a resolution of $227 \times 227$ pixels.

To overcome the adverse effect of overfitting, all of the dataset packages are reorganized. As a result, a reliable and effective decision-making performance for classifying the infected people instances is achieved. The small batch size is set to 36 in the training phase, and all images are normalized using the mean subtracting procedure. The average computational time for the proposed SQNet model on CPU is $0.347 \mathrm{~s}$ and for GPU it is $0.152 \mathrm{~s}$. Cumulatively, there are a total of 1502 healthy cases, 1463 bacterial cases(non-CorV), 2513 viral cases, and $84 \mathrm{CorV}$ patient cases. In pneumonia cases, both viral and bacterial samples are considered. Table 4 depicts the raw datasets and augmented datasets.

The deep SQNet model proposed incorporates optimization in the training stage as well as a validation step. 


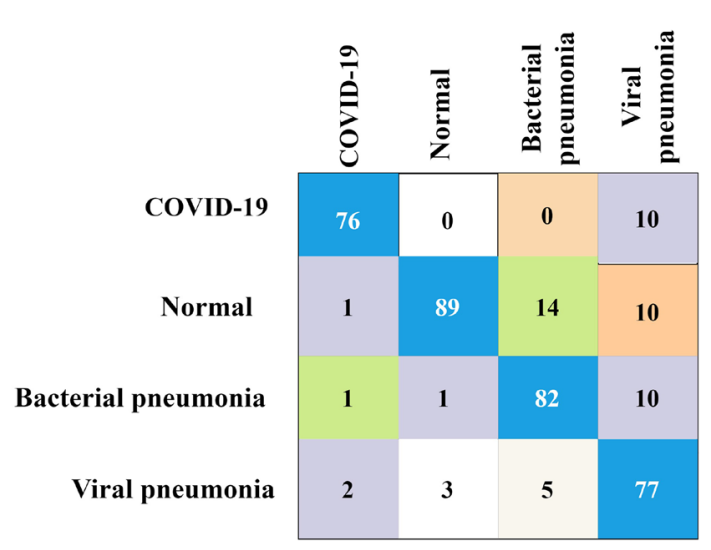

Fig. 6 Confusion matrix for 4-class classification

Figure 5 shows the optimization process's objective function. Because of the model saturation, function evaluation is shown to terminate after 30 iterations can be seen. The minimal observed aim to create the best model is accomplished at the end of the 9th iteration. The augmentation approach increases the performance enhancement by approximately 20 times for the proposed model.

The best model parameters obtained during the training procedure are utilized in the proposed model. The relevant dataset is used for the pre-training and testing operations from the trained and test packages. The current study presents the dataset results to evaluate the performance of the augmentation approach.

\subsubsection{Results}

The confusion matrix of the test procedure of the proposed model is depicted in Fig. 6. In the confusion matrix, each column represents the accurate value of each class and the

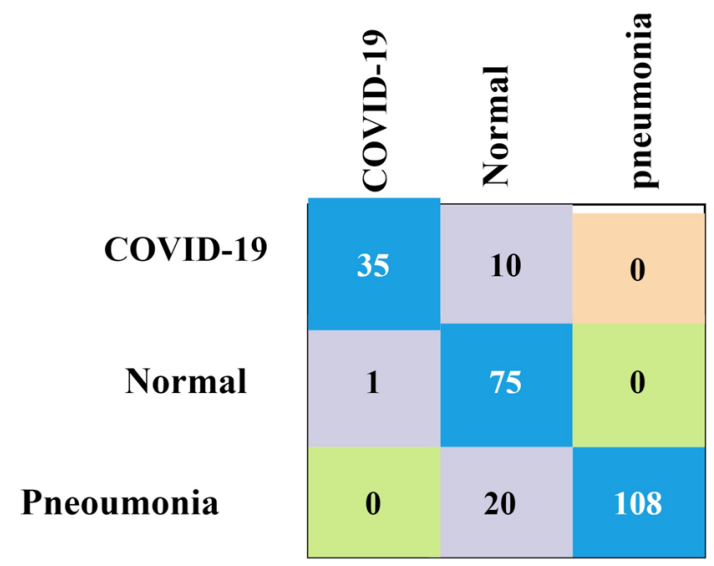

Fig. 7 Confusion matrix after fine-tuned into three-class

row states the individual accurate value of the class. Figure 6 depicts the confusion matrix of 4-class of Cov-dataset2. The average ACR, TR, FLT, SPFC, F-measure, and MCR are described in Table 5. In such a case, the accuracy is $97.8 \%$, the value of specificity is $91.0 \%$, and f-measure is $92 \%$ of the SQNet model. Figure 10a-d depicts the pictorial representation of the proposed model.

The performance for pneumonia (bacterial and viral) is less compared to other classes. When it combines into a single class, the average accuracy increases significantly by making the slight modification in 4-class with the SQNet model and fine-tuned. The model was tested on $45 \mathrm{CorV}$ cases, 76 Normal, and 128 pneumonia cases after the finetuning process. The confusion matrix for three-class is described in Fig. 7. After combining pneumonia classes, the average Accuracy of the SQNet model is increased from $97.8 \%$ to $98.4 \%$ as shown in Table 6.

Figure 8 depicts the graphical representation accuracy of the proposed model.
Table 5 Average accuracy, precision, recall, F-measure, specificity (in \%)

\begin{tabular}{lllllll}
\hline Classes & ACR & TR & FLT & SPFC & F-measure & MCR \\
\hline CorV & 96.71 & 96.2 & 94.13 & 99.6 & 95.12 & 62.7 \\
Normal & 98.6 & 93.4 & 98.52 & 97.4 & 96.71 & 58.09 \\
Bacterial pneumonia & 97.5 & 87.21 & 86.7 & 94.1 & 88.51 & 56.11 \\
Viral pneumonia & 97.7 & 86.1 & 83.2 & 94.3 & 88.3 & 56.2 \\
Average & 97.8 & 92.7 & 91 & 96 & 92.7 & 56.0 \\
\hline
\end{tabular}

Table 6 Average Accuracy, Precision, Recall, F-measure, Specificity(in \%) (three-class classification)

\begin{tabular}{lllllll}
\hline Classes & ACR & TR & FLT & SPFC & F-measure & MCR \\
\hline CorV & 99.22 & 98.1 & 88.9 & 94.0 & 98.5 & 71.8 \\
Normal & 98.7 & 93.2 & 86.51 & 90.1 & 97.41 & 58.09 \\
Pneumonia & 97.52 & 91.2 & 93.4 & 91.3 & 89.51 & 56.11 \\
Average & 98.4 & 94.2 & 89.6 & 91.8 & 95.1 & 61.9 \\
\hline
\end{tabular}


Fig. 8 Accuracy of the proposed model
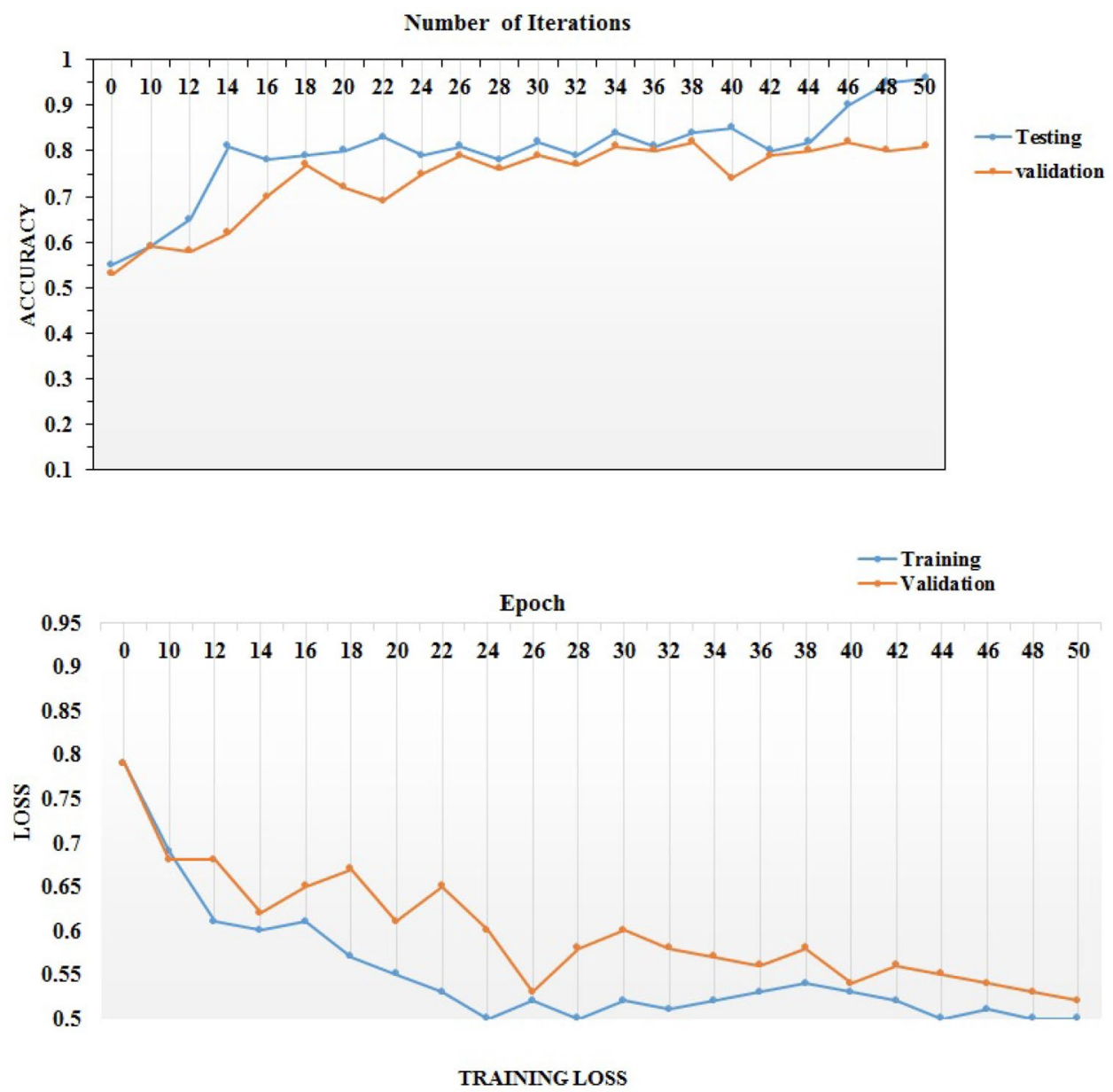

Table 6 represents that SQNet has a $92.7 \%$ precision value. The F-measure and MCR values also show that a stable categorization has been achieved. A higher recall value indicates that a low number of false negatives (FLN) is an encouragement to better outcomes.

\subsection{Comparative analysis}

On February 11, 2020 WHO declared the CorV illness to be an epidemic. The identification of CorV has become a global menace, as a result of it being declared a pandemic. The use of DL techniques in images classification procedures can aid in the early detection of illness. In terms of the deep neural network, $\mathrm{CNN}$ performs better than the traditional diagnosis methods for efficient classification. Deep SQNet is presented as a fast, reliable, and efficient CorV diagnostic technique in the current study. The suggested approach classifies CT images into Healthy, Pneumonia, viral, and CorV categories. $\mathrm{Li}$ et al. (2020) present a COVID-Xpert architecture based on DenseNet using medical images for identifying CorV patients. The experiment results have shown the overall accuracy of $89.8 \%$ via transfer learning. Wang et al. (2020) presents the COVID-Net architecture for CorV diagnosis, and the primary model based on the customized CNN. The model architecture is improved via machine-driven design. Experiment results provide overall accuracy, COM, and COR scores of $0.923,0.887$, and 0.913 , respectively. The COVIDx dataset utilized is also shared and collected by the authors. Farooq and Hafeez (2020) proposed a ResNet-based system for identification of CorV. The model's accuracy has remained constant at 0.962 . Table 7 depicts that the proposed SQNet model has achieved a higher accuracy value of 98.4\% when compared to other CNN based models.

\subsubsection{Discussion}

Since the publication of a dataset generated by Cohen, researchers have been studying chest X-ray pictures for precise prediction of CorV infection (Cohen et al. 2020). Moreover, numerous studies have been attempted to create an accurate diagnostic model by utilizing DL methods. The TL method has been widely used in CNN-based networks. On the other hand, most of the researchers had performed experiments with a restricted amount of data. In certain situations, the datasets are also unbalanced. For such purposes, the pre-defined CNN models such as 


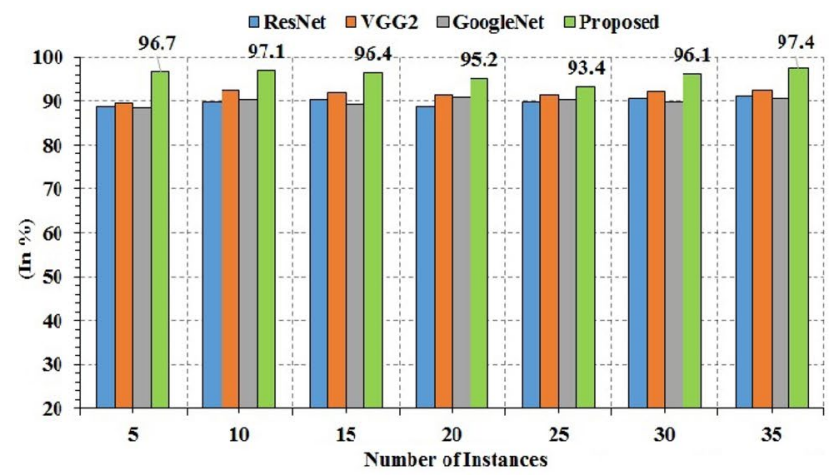

(a) Accuracy

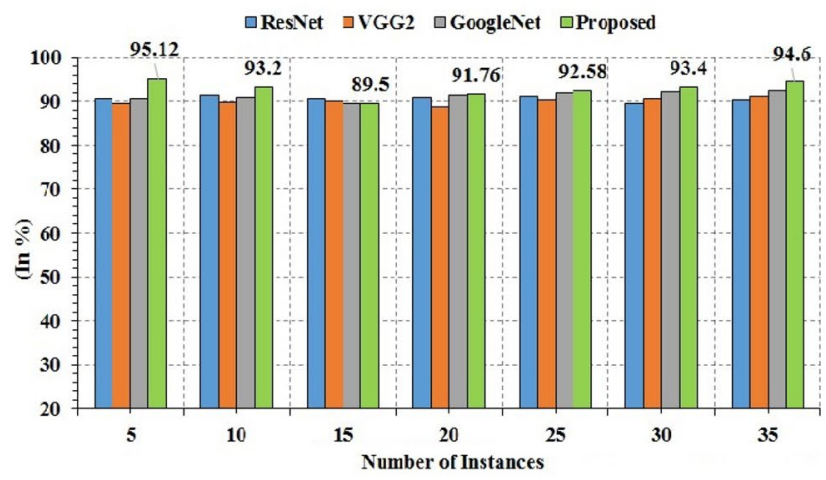

(c) F-measure

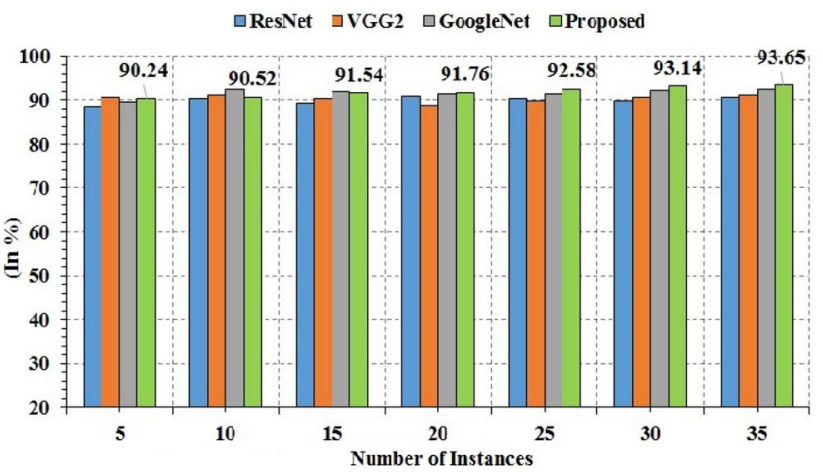

(b) Specificity

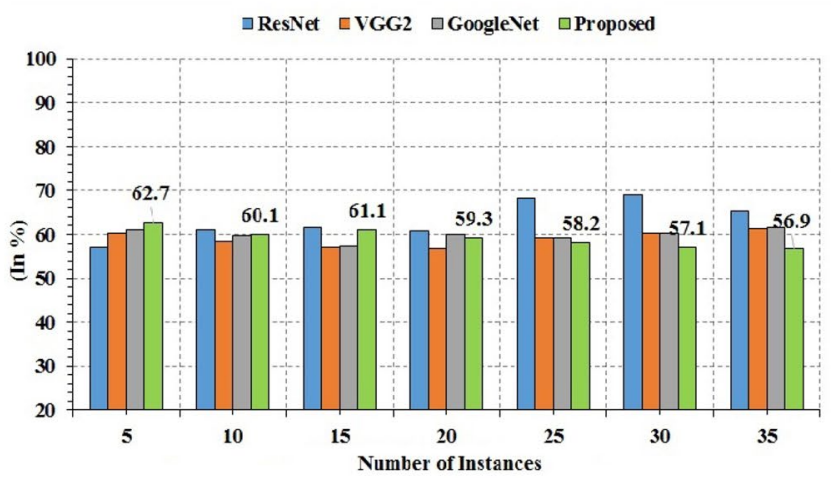

(d) MCR

Fig. 10 a Accuracy, b Specificity, c F-measure, d Matthew correlation coefficient (MCR)

Table 7 Comparative analysis

\begin{tabular}{|c|c|c|c|c|c|c|c|}
\hline Metrics/Authors & Class & Methods & Datasets & $\begin{array}{l}\text { Augmentation/Fine- } \\
\text { tuning Technique }\end{array}$ & SPEC & SEN & Accuracy(in\%) \\
\hline Afshar et al. (2020) & 4 & CapsuleNet & Public & Fine-tuned & 96 & 90.1 & 95.7 \\
\hline Song et al. (2021) & 2 & DRE-Net & Private & Tranfer learning & - & 93 & 98.5 \\
\hline Li et al. (2020) & 3 & DenseNet & Repository & No & 92 & 87 & 88.9 \\
\hline $\begin{array}{l}\text { Apostolopoulos and Mpesi- } \\
\text { ana (2020) }\end{array}$ & 2 & VGG-19 & Public & Tranfer learning & 96.2 & - & 93.2 \\
\hline Ozturk et al. (2020) & 3 & DarkNet & Public & No & 90 & 92.1 & 87.1 \\
\hline Hariri and Narin (2021) & 3 & sgdm & Public & Tranfer learning & - & - & 98 \\
\hline Das et al. (2020) & 3 & Xception & Public & Transfer learning & 96.9 & 97.1 & 97.4 \\
\hline Wang et al. (2020) & 2 & ResNet50 & Public & Tranfer learning & 92.1 & 91 & 97.8 \\
\hline Narin et al. (2021) & 2 & nCoVNet50 & Public & Tranfer learning & 89.1 & 98.0 & 88.8 \\
\hline Proposed System & 4 & SQNet & Repository & Both & 96.0 & 91 & 98.4 \\
\hline
\end{tabular}

AlexNet, VGG2, ResNet52, GoogleNet, AlexNet, and InceptionV3 for the prediction of CorV infections are evaluated. The datasets are collected from two sources cohen and "COVIDchest Xray-dataset" (Cohen et al. 2020). The experiment is evaluated on a large dataset by taking into several factors to enhance the performance of the proposed model for automated diagnosing CorV. Furthermore, the advantage to use the fine-tuned SQNet model, it uses fewer parameters. In addition, transfer learning is used to find the solution to the overfitting problem. The experimental results have shown that the SQNet model achieved 98.4\% accuracy as compared to other models. The present approach is cost-effective and can assist in making timely decisions. The main goal of the present study is to take effective treatment decisions for quarantine patients, which will assist to reduce the spread of CorV infection. 
In the future, the current research intends to validate the proposed model by incorporating more images.

\section{Conclusion}

In the present work, a DL-based model is proposed for effectively classifying the CorV infection cases from healthy, viral, and bacterial pneumonia cases by using chest X-ray images. The model is developed to provide accurate diagnostics for both binary and multi-class classification. The proposed model produced $97.8 \%$ and 98.4\% accuracy for four-class and three-class classification respectively. The limitation of the current research is the inability of the patient for X-ray scanning in a severe situation. In the future, the presented model will be placed in a cloud to provide diagnosis instantly and to help affected patients immediately. The current research intends to make a model more robust and accurate for the future. The current research contributes to the development of cost-effective approaches for combating the disease. Such approaches might be explored for further research to demonstrate real-world implementation.

\section{Funding None}

Data availability The data used to support the findings of the present study is included within the article.

\section{Declarations}

Conflict of interest Authors have no conflict of interest.

Ethics approval and consent to participate The article does not contain any studies with human participants or animals performed by any of the authors.

\section{References}

Abdani SR, Zulkifley MA, Zulkifley NH ( 2020) A lightweight deep learning model for COVID-19 detection. In: 2020 IEEE Symposium on Industrial Electronics Applications (ISIEA), IEEE, pp 1-5

Adair LB II, Ledermann EJ (2020) Chest ct findings of early and progressive phase COVID-19 infection from a us patient. Radiol Case Rep 15(7):819-824

Afshar P, Heidarian S, Naderkhani F, Oikonomou A, Plataniotis KN, Mohammadi A (2020) Covid-caps: a capsule network-based framework for identification of COVID-19 cases from X-ray images. Pattern Recogn Lett 138:638-643

Ahmed S, Hossain MF, Noor MBT ( 2021) Convid-net: An enhanced convolutional neural network framework for COVID-19 detection from X-ray images. In: Proceedings of International
Conference on Trends in Computational and Cognitive Engineering, Springer, pp 671-681

Albawi S, Mohammed T.A, Al-Zawi S ( 2017) Understanding of a convolutional neural network. In: 2017 International Conference on Engineering and Technology (ICET). Ieee, pp. 1-6

Alqudah A.M, Qazan S, Alqudah A ( 2020) Automated systems for detection of COVID-19 using chest X-ray images and lightweight convolutional neural networks

Apostolopoulos ID, Mpesiana TA (2020) Covid-19: automatic detection from X-ray images utilizing transfer learning with convolutional neural networks. Phys Eng Sci Med 43(2):635-640

Bai L, Yang D, Wang X, Tong L, Zhu X, Zhong N, Bai C, Powell CA, Chen R, Zhou J et al (2020) Chinese experts' consensus on the internet of things-aided diagnosis and treatment of coronavirus disease 2019 (COVID-19). Clin eHealth 3:7-15

Bailer C, Habtegebrial T, Stricker D et al (2018) Fast feature extraction with cnns with pooling layers. arXiv preprintarXiv:1805.03096

Brunetti A, Carnimeo L, Trotta GF, Bevilacqua V (2019) Computerassisted frameworks for classification of liver, breast and blood neoplasias via neural networks: A survey based on medical images. Neurocomputing 335:274-298

Chan Y-H, Zeng Y-Z, Wu H-C, Wu M-C, Sun H-M (2018)Effective pneumothorax detection for chest $\mathrm{X}$-ray images using local binary pattern and support vector machine. J Healthcare Eng 2018

Chang D, Lin M, Wei L, Xie L, Zhu G, Cruz CSD, Sharma L (2020) Epidemiologic and clinical characteristics of novel coronavirus infections involving 13 patients outside wuhan, china. JAMA 323(11):1092-1093

Chang X, Nie F, Wang S, Yang Y, Zhou X, Zhang C (2015) Compound rank- $k$ projections for bilinear analysis. IEEE Trans Neural Networks Learning Systems 27(7):1502-1513

Chouhan V, Singh SK, Khamparia A, Gupta D, Tiwari P, Moreira C, Damaševičius R. De, Albuquerque VHC (2020) A novel transfer learning based approach for pneumonia detection in chest X-ray images. Appl Sci 10(2):559

Cohen J.P, Morrison P, Dao L, Roth K, Duong T.Q, Ghassemi M ( 2020) Covid-19 image data collection: Prospective predictions are the future. arXiv preprintarXiv:2006.11988

Corman VM, Landt O, Kaiser M, Molenkamp R, Meijer A, Chu DK, Bleicker T, Brünink S, Schneider J, Schmidt ML et al (2020) Detection of 2019 novel coronavirus (2019-ncov) by real-time rt-pcr. Eurosurveillance 25(3):2000045

Das N.N, Kumar N, Kaur M, Kumar V, Singh D ( 2020) Automated deep transfer learning-based approach for detection of COVID-19 infection in chest $\mathrm{X}$-rays. Irbm

Dorj U-O, Lee K-K, Choi J-Y, Lee M (2018) The skin cancer classification using deep convolutional neural network. Multimedia Tools Appl 77(8):9909-9924

Farooq M, Hafeez A ( 2020) Covid-resnet: A deep learning framework for screening of COVID19 from radiographs. arXiv preprintarXiv:2003.14395

Gaál G Maga B, Lukács A ( 2020) Attention u-net based adversarial architectures for chest X-ray lung segmentation. arXiv preprintarXiv:2003.10304

Gorbalenya AE, Baker SC, Baric R, Groot RJd, Drosten C, Gulyaeva AA, Haagmans BL, Lauber C, Leontovich AM, Neuman BW et al (2020) Severe acute respiratory syndrome-related coronavirus: the species and its viruses-a statement of the coronavirus study group

Hariri W, Narin A (2021) Deep neural networks for COVID-19 detection and diagnosis using images and acoustic-based techniques: a recent review. Soft Comput 2:1-18

Hemdan EED, Shouman MA, Karar ME ( 2020) Covidx-net: A framework of deep learning classifiers to diagnose COVID-19 in X-ray images. arXiv preprintarXiv:2003.11055 
Huang J.-H, Yang C.-H.H, Liu F, Tian M, Liu Y.-C, Wu T.-W, Lin I, Wang K, Morikawa H, Chang H et al (2021) Deepopht: medical report generation for retinal images via deep models and visual explanation. In: Proceedings of the IEEE/CVF winter conference on applications of computer vision, pp. 2442-2452

Iandola F. N, Han S, Moskewicz M.W, Ashraf K, Dally W.J, Keutzer K (2016) Squeezenet: Alexnet-level accuracy with 50x fewer parameters and $<0.5 \mathrm{mb}$ model size. arXiv preprintarXiv: 1602.07360

Jain R, Gupta M, Taneja S, Hemanth DJ (2021) Deep learning based detection and analysis of COVID-19 on chest X-ray images. Appl Intell 51(3):1690-1700

Lai C-C, Shih T-P, Ko W-C, Tang H-J, Hsueh P-R (2020) Severe acute respiratory syndrome coronavirus 2 (sars-cov-2) and coronavirus disease-2019 (COVID-19): The epidemic and the challenges. Int J Antimicrob Agents 55(3): 105924

Li Q, Guan X, Wu P, Wang X, Zhou L, Tong Y, Ren R, Leung KS, Lau EH, Wong JY et al (2020) Early transmission dynamics in Wuhan, China, of novel coronavirus-infected pneumonia. New Engl J Med 2:2

Li X, Li C, Zhu D ( 2020) Covid-mobilexpert: On-device COVID-19 screening using snapshots of chest X-ray. arXiv preprintarXiv:2004.03042

Li Z, Nie F, Chang X, Nie L, Zhang H, Yang Y (2018) Rank-constrained spectral clustering with flexible embedding. IEEE Trans Neural Netw Learn Syst 29(12):6073-6082

Li Z, Nie F, Chang X, Yang Y, Zhang C, Sebe N (2018) Dynamic affinity graph construction for spectral clustering using multiple features. IEEE Trans Neural Netw Learn Syst 29(12):6323-6332

Li Z, Yao L, Chang X, Zhan K, Sun J, Zhang H (2019) Zero-shot event detection via event-adaptive concept relevance mining. Pattern Recogn 88:595-603

Liu X, Faes L, Kale AU, Wagner SK, Fu DJ, Bruynseels A, Mahendiran T, Moraes G, Shamdas M, Kern C et al (2019) A comparison of deep learning performance against health-care professionals in detecting diseases from medical imaging: a systematic review and meta-analysis. Lancet Digital Health 1(6):e271-e297

Loey M, Smarandache FM, Khalifa NE (2020) Within the lack of chest COVID-19 X-ray dataset: a novel detection model based on gan and deep transfer learning. Symmetry 12(4):651

Luo M, Chang X, Nie L, Yang Y, Hauptmann AG, Zheng Q (2017) An adaptive semisupervised feature analysis for video semantic recognition. IEEE Trans Cybern 48(2):648-660

Mahase E ( 2020) Coronavirus: COVID-19 has killed more people than sars and mers combined, despite lower case fatality rate

Mahmud T, Rahman MA, Fattah SA (2020) Covxnet: A multi-dilation convolutional neural network for automatic COVID-19 and other pneumonia detection from chest X-ray images with transferable multi-receptive feature optimization. Comput Biol Med 122:103869

Narin A, Kaya C, Pamuk Z ( 2021) Automatic detection of coronavirus disease (COVID-19) using X-ray images and deep convolutional neural networks. Pattern Anal Appl, pp 1-14

Nayak SR, Nayak DR, Sinha U, Arora V, Pachori RB (2021) Application of deep learning techniques for detection of COVID-19 cases using chest X-ray images: a comprehensive study. Biomed Signal Process Control 64:102365

Ozturk T, Talo M, Yildirim EA, Baloglu UB, Yildirim O, Acharya UR (2020) Automated detection of COVID-19 cases using deep neural networks with X-ray images. Comput Biol Med 121:103792

Pahwa E, Mehta D, Kapadia S, Jain D, Luthra A ( 2021) Medskip: Medical report generation using skip connections and integrated attention, In: Proceedings of the IEEE/CVF International Conference on Computer Vision, pp 3409-3415
Panwar H, Gupta P, Siddiqui MK, Morales-Menendez R, Singh V (2020) Application of deep learning for fast detection of COVID19 in X-rays using ncovnet. Chaos Solitons Fractals 138:109944

Ren P, Xiao Y, Chang X, Huang P-Y, Li Z, Chen X, Wang X (2021) A comprehensive survey of neural architecture search: challenges and solutions. ACM Comput Surv (CSUR) 54(4):1-34

Salman S, Salem ML (2020) Routine childhood immunization may protect against COVID-19. Med Hypotheses 140:109689

Sethy PK, Behera SK (2020) Detection of coronavirus disease (COVID-19) based on deep features

Shibly KH, Dey SK, Islam MT-U, Rahman MM (2020) Covid faster r-cnn: A novel framework to diagnose novel coronavirus disease (COVID-19) in X-ray images. Inform Med Unlocked 20:100405

Song Y, Zheng S, Li L, Zhang X, Zhang X, Huang Z, Chen J, Wang R, Zhao H, Zha Y et al ( 2021) Deep learning enables accurate diagnosis of novel coronavirus (COVID-19) with ct images. IEEE/ ACM Trans Comput Biol Bioinform

Stoecklin SB, Rolland P, Silue Y, Mailles A, Campese C, Simondon A, Mechain M, Meurice L, Nguyen M, Bassi C et al (2020) First cases of coronavirus disease 2019 (COVID-19) in France: surveillance, investigations and control measures, January 2020. Eurosurveillance 25(6):2000094

Swati ZNK, Zhao Q, Kabir M, Ali F, Ali Z, Ahmed S, Lu J (2019) Brain tumor classification for $\mathrm{mr}$ images using transfer learning and fine-tuning. Comput Med Imaging Graph 75:34-46

Ucar F, Korkmaz D (2020) Covidiagnosis-net: deep bayes-squeezenet based diagnosis of the coronavirus disease 2019 (COVID-19) from X-ray images. Med Hypotheses 140:109761

Wang L, Lin ZQ, Wong A (2020) Covid-net: A tailored deep convolutional neural network design for detection of COVID-19 cases from chest X-ray images. Sci Rep 10(1):1-12

Wang W, Xu Y, Gao R, Lu R, Han K, Wu G, Tan W (2020) Detection of sars-cov-2 in different types of clinical specimens. JAMA 323(18): 1843-1844

Xie X, Zhong Z, Zhao W, Zheng C, Wang F, Liu J (2020) Chest ct for typical coronavirus disease 2019 (COVID-19) pneumonia: relationship to negative rt-pcr testing. Radiology 296(2):E41-E45

Xu X, Jiang X, Ma C, Du P, Li X, Lv S, Yu L, Ni Q, Chen Y, Su J et al (2020) A deep learning system to screen novel coronavirus disease 2019 pneumonia. Engineering 6(10):1122-1129

Yadav SS, Jadhav SM (2019) Deep convolutional neural network based medical image classification for disease diagnosis. J Big Data 6(1):1-18

Yildirim O, Talo M, Ay B, Baloglu UB, Aydin G, Acharya UR (2019) Automated detection of diabetic subject using pre-trained $2 \mathrm{~d}-\mathrm{cnn}$ models with frequency spectrum images extracted from heart rate signals. Comput Biol Med 113:103387

Yu E, Sun J, Li J, Chang X, Han X-H, Hauptmann AG (2018) Adaptive semi-supervised feature selection for cross-modal retrieval. IEEE Trans Multimedia 21(5):1276-1288

Zhang H-T, Zhang J-S, Zhang H-H, Nan Y-D, Zhao Y, Fu E-Q, Xie Y-H, Liu W, Li W-P, Zhang H-J et al (2020) Automated detection and quantification of COVID-19 pneumonia: Ct imaging analysis by a deep learning-based software. Eur J Nucl Med Mol Imaging 47(11):2525-2532

Zhou R, Chang X, Shi L, Shen Y-D, Yang Y, Nie F (2019) Person reidentification via multi-feature fusion with adaptive graph learning. IEEE Trans Neural Netw Learn Syst 31(5):1592-1601

Publisher's Note Springer Nature remains neutral with regard to jurisdictional claims in published maps and institutional affiliations. 